\title{
Evaluation of the effects of estradiol on the hypothalamic-pituitary-adrenal axis response during systemic and local inflammation
}

\author{
Nivin Sharawy ${ }^{1 *}$, Mohamed Hassan ${ }^{1}$, Laila Rashed ${ }^{2}$, Wael Shawky ${ }^{3}$, Moshira Rateb ${ }^{1}$ \\ ${ }^{1}$ Department of Physiology, Kasr EL Eini University, Cairo, Egypt; * Corresponding Author: n_sharawy@yahoo.com \\ ${ }^{2}$ Department of Biochemistry, Kasr EL Eini University, Cairo, Egypt \\ ${ }^{3}$ Department of Pathology, Kasr EL Eini University, Cairo, Egypt
}

Received 20 June 2012; revised 15 July 2012, accepted 13 August 2012

\section{ABSTRACT}

The immune and nervous systems are closely interrelated through their messenger molecules: cytokines, hormones and nitric oxide. There are sex differences in the magnitude of the inflammatory response and one of the mechanism by which female sex steroids modulate the inflammatory response could be through the hypothalamo-pituitary-adrenal axe (HPA axes). In this study, we aimed to investigate the inter-relationship between the different levels of estradiol hormone and the different inflammatory stimuli on the HPA axis response, and the role of interleukin 6 (IL6) and nitric oxide (NO). This was achieved by using 100 female albino rats divided in to 3 main groups: The first group was the sham group, which was used to test the effect of all sham procedures used in the study. The two other groups represented the two inflammatory states: local (induced by formaline injection) and systemic (cecal ligation and puncture: CLP). Under each inflammatory state, the effect of intact ovary, estradiol treated ovariectomy and placebo treated ovariectomy were studied. HPA responses were evaluated, 24 hours after the sham or the inflammatory procedure, through the measurement of the plasma levels of Corticotropin-releasing Hormone (CRH), adrenocorticotropes Hormone (ACTH) and cortisol. Both IL6 and nitrite were also measured.We found that high physiological levels of estradiol reduced the enhanced HPA response induced by different inflammatory mediators during systemic and local inflammation. With significant enhancement of the HPA response observed mainly during local inflammation. We conclude that both the levels of estradiol hormone and the type of inflammation seem to play an important determining role on HPA response. IL6 and NO play important roles that could explain the previous findings.

Keywords: Formalin; CLP; Estradiol; Ovariectomy; HPA; Inflammation

\section{INTRODUCTION}

Despite recent advances in medicine, Systemic Inflammatory Response Syndrome (SIRS) remains one of the leading immediate causes of death in critically ill patients [1-3].

The acute phase and chronic phase of SIRS are very different in terms of the metabolic and endocrine responses[4]. The metabolic adaptations result in an increased availability of glucose, free fatty acids and amino acids as substrates for vital organs such as the immune system. These changes have consistently been considered to be adaptive and beneficial, as they may postpone anabolism and, at the same time, activate the immune response [5]. In the last decade, many efforts have been made to further understand the neuroendocrine characteristics of stress and it has appeared that the acute phase is mainly characterised by an actively secreting anterior pituitary gland. While this has been documented for all hypothalamic-pituitary-dependent axes, the focus of our re-search was on HP adrenal cortex axes, which is the site of action that brought about biochemical changes in response to severe stress with the release of corticotrophin-releasing hormone $(\mathrm{CRH})$ from the hypothalamus and activation of the adreno corticotrophic hormone (ACTH) in the anterior pituitary, which moves to the adrenal cortex and stimulates the production of cortisol [6]. The previous studies clearly implicate the role of HPA as immunoregulator during different stress stimuli, whether inflammatory, traumatic or psychological, and that cytokines and other humoral mediators of inflammation are potent activators of the central stress response, 
constituting the afferent limb of a feedback loop through which the immune/inflammatory system and the CNS communicate $[7,8]$.

Gender differences in the incidence and lethality of SIRS have been reported $[9,10]$.

However, the controversial results of the previous studies addressing the gender differences in stress induced HPA response could be very confusing making it difficult to clearly identify the role of sex hormones in the pathophysiology of SIRS [7,10-20].

Our aim was to investigate the effects of the different estradiol levels and the type of stress on HPA response, and the role of centrally (brain and pituitary) and peripherally formed IL6 and the centrally formed nitric oxide on the stress induced HPA response.

\section{MATERIALS AND METHODS}

\subsection{Animals}

After obtaining approval from the Kasr El Aini Animal Care Committee, we used 100 female Lewis rats from the animal house in Kasr El Aini faculty of medicine for the experiments (body weight: $220-240 \mathrm{~g}$ ). The animals were housed in chip-bedded cages and, prior to experiments, acclimated for 1 week in the air conditioned institutional animal care unit. They were housed under 12 hour light/dark cycles, with free access to water and standard rat chow.

\subsection{Experimental Groups}

Female albino rats were divided in to 3 main groups: group I. Sham female rats $(\mathrm{n}=40)$, which were sub-divided in to 2 main subgroups: subgroup Ia. Sham cecal ligation and puncture (sham CLP) $(\mathrm{n}=20)$, which was divided in to 2 types: $\mathrm{Ia}_{1}$ without sham ovariectomy (-sham OVX); $\mathrm{Ia}_{2}$ with sham ovariectomy (+sham OVX), and subgroup Ib. Sham local inflammation (sham LI)(n= 20 ), which was also di-vided in to 2 types: $\mathrm{Ib}_{1}$ without sham ovariectomy (-sham OVX); $\mathrm{Ib}_{2}$ with sham ovariectomy $(+$ sham OVX). Female rats in group II $(n=30)$ were subjected to cecal ligation and puncture (CLP), and subdivided in to 3 main subgroups: subgroup IIa female rats with intact ovary and subjected to CLP (CLP) (n = 10), subgroup IIb. ovariectomized female rats received placebo treatment sesame oil replacement (Misr CO. for pharm. Ind., Cairo, Egypt) $(\approx 500 \mu \mathrm{l} \mathrm{SC}$ each day) 3 weeks before subjection to cecal ligation and puncture $(\mathrm{OVX}+$ oil + CLP $)(\mathrm{n}=10)$ and subgroup IIc. Ovariectomized female rats received estradiol replace-ment (Misr CO. for pharm. Ind., Cairo, Egypt) $4 \mu \mathrm{g} / 100 \mathrm{~g}$ body weight of estradiol benzoate (EB) in sesame oil SC each day for 3 weeks before induction of cecal ligation and puncture $(\mathrm{OVX}+\mathrm{E} 2+\mathrm{CLP})(\mathrm{n}=10)$. Female rats in group III $(\mathrm{n}=30)$ were subjected to local inflammation
(LI), and subdivided in to 3 main subgroups: subgroup IIIa. Female rats with intact ovary and subjected to local inflammation (LI) $(\mathrm{n}=10)$, subgroup IIIb: ovariectomized female rats received placebo treatment sesame oil replacement (Misr CO. for pharm. Ind., Cairo, Egypt) $(\approx 500 \mu \mathrm{l} \mathrm{SC}$ each day) 3 weeks before subjection to Local inflammation $(\mathrm{OVX}+$ oil + LI) $(\mathrm{n}=10)$ and subgroup IIIc. ovariectomized female rats received estradiol replacement :-(Misr CO. for pharm. Ind., Cairo, Egypt) 4 $\mu \mathrm{g} / 100 \mathrm{~g}$ body weight of estradiol benzoate (EB) in sesame oil SC each day for 3 weeks before induction of Local inflammation $(\mathrm{OVX}+\mathrm{E} 2+\mathrm{LI})(\mathrm{n}=10)$. The dose of estradiol treatment was chosen according to Suzuki and Handa [21].

\subsection{General Protocol}

The sham ovariectomized female rats were subjected only to a midline ventral laparotomy 3 weeks before sham CLP or sham LI. In the ovariectomized female rats the bilateral ovaries were located and excised through a midventral incision under anesthesia with ketamine (100 $\mathrm{mg} / \mathrm{kg}$ ) (Amoun pharmaceutical Co. for pharm Ind., Cairo, Egypt) and xylazine $(2.5 \mathrm{mg} / \mathrm{kg}$ ) (Amoun pharmaceutical Co. for pharm Ind., Cairo, Egypt) intra-peritoneal (i.p.) $[22,23]$.

The cecal ligation and puncture (CLP) was carried out under anesthesia with ketamine $(100 \mathrm{mg} / \mathrm{kg})$ and $\mathrm{xy}-$ lazine $(2.5 \mathrm{mg} / \mathrm{kg}$ ) (Amoun pharmaceutical Co. for pharm Ind., Cairo, Egypt) i.p. After shaving and disinfection, a 2-cm-long median laparotomy was performed above the symphysis with exposure and isolation of the cecum. The cecum was tied off $1 \mathrm{~cm}$ from the end and punctured with a single hole by using a 21-gauge needle. Within the sham CLP animals were only subjected to a midline ventral laparotomy and cecum manipulation [22].

The local inflammation (LI) was carried out under anesthesia with ketamine $(100 \mathrm{mg} / \mathrm{kg}$ ) (Amoun pharmaceutical Co. for pharm Ind., Cairo, Egypt) and xylazine (2.5 $\mathrm{mg} / \mathrm{kg}$ ) (Amoun pharmaceutical Co. for pharm Ind., Cairo, Egypt) i.p., after which animals received an intraplantar injection of $0.1 \mathrm{ml} \mathrm{5 \%}$ formalin freshly diluted in $0.9 \%$ isotonic sterile saline in the right hind paw. In sham LI, the female rats were injected with normal saline $(0.2$ $\mathrm{ml}$ ) on the right hind paw [24] (Figure 1).

Twenty four hours after induction of either cecal ligation and puncture or local inflammation, blood samples were taken to measure the plasma levels of estradiol, $\mathrm{CRH}, \mathrm{ACTH}$, cortisol and IL6. After which the rats were decapitated and the parietal bone were cut out with scissors, whole brain tissue was gently removed, the optic chiasma was located as land mark for pituitary gland, which lies in sella turcica. Both brain and pituitary tissues were frozen separately for further homogenous and chemical measurements of IL6. 


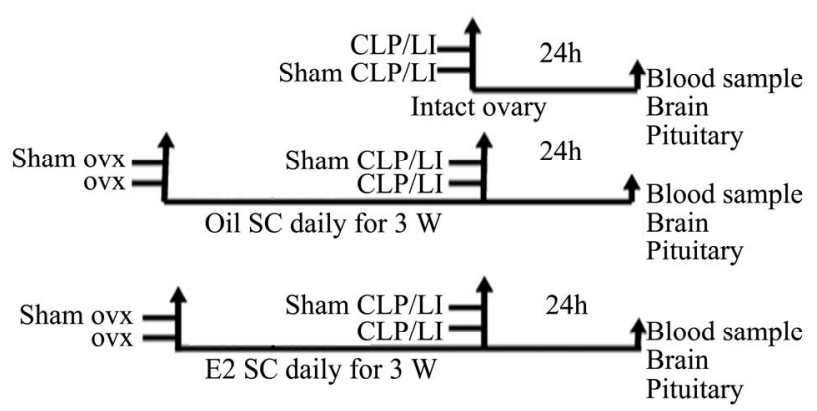

Figure 1. General experimental protocol for female rats with intact ovary and subcutaneous (SC) oil and esradiol (E2) treated ovariectomized (OVX) female rats 3 weeks before subjection to cecal ligation and puncture (CLP) and local inflammation (LI). Blood sample, brain and pituitary tissues were taken 24 hours after CLP and LI.

\subsection{Tissue Homogenization}

A portion of the brain and pituitary tissues were homogenized for testing in IL6. Homogenization was performed after the tissue samples had been diluted in 5 volume of homogenate buffer [10 mM HEPES ( $\mathrm{pH} 7.9$ ), $10 \mathrm{mM} \mathrm{KCL}, 0.1 \mathrm{mM}$ EGTA, $1 \mathrm{mM}$ DTT, and $0.5 \mathrm{mM}$ phenylmethanesulfonyl fluoride] (all reagents were purchased from Sigma-Aldrich, St. Louis, MO, USA )using a vertishear tissue homogenizer. Brain and pituitary homogenates were centrifuged at $3000 \mathrm{~g}$ for $15 \mathrm{~min}$ at $4^{\circ} \mathrm{C}$. The supernatants were subsequently stored at $-80^{\circ} \mathrm{C}$.

\subsection{Measurement of IL6}

Venous blood samples were collected from all rats; plasma was separated and stored at $-70^{\circ} \mathrm{C}$ until analysis. Time of sampling was between (12:00-13:00 P.M.) Commercially available ELISA kits provided by USCNLIFE Company; Wuhan 430079, CHINA were used for hormonal estimations [estradiol, $\mathrm{CRH}, \mathrm{ACTH}$ and cortisol] and ELISA kits provided by camarillo; California 93012, USA were used for IL6 measurement in the plasma and supernatants obtained by brain tissue homogenization

\subsection{Measurement of Nitrite}

We used the Griess Reagent System to measure brain and pituitary nitrite $\left(\mathrm{NO}_{2}^{-}\right)$, which is one of two primary stable and nonvolatile breakdown products of nitric oxide (NO), by using the commercial available colorimetric assay kit supplied by Alexis Biochemicals Company, San Diego, CA. 92121 United States.

\subsection{Statistical Analysis}

The results are given as means \pm standard deviation (SD). Results were analyzed by using the software Prism 5 (GraphPad Software, La Jolla, CA, USA). First, data were tested for normal distribution by using the Kolmogorov-Smirnov test. If normal distribution was established, one-way analysis of variance (ANOVA) was performed. If significant differences appeared, a post hoc analysis with the Newman-Keuls multiple comparison test was conducted. The investigations of values in multifactorial design were examined by means of two-way analysis of variance (two-way repeated-measures ANOVA). A value of $p<0.05$ was considered statistically significant.

\section{RESULTS}

\subsection{The Plasma Levels of Estradiol, CRH, ACTH and Cortisol}

In comparison to the corresponding sham subgroups, we found that both non ovariectomized and ovariectomized female rats subjected to either CLP and LI showed significant decrease $(\mathrm{p}<0.05)$ in the level of estradiol with significant increases $(p<0.05)$ in the levels of CRH, ACTH and cortisol. [CLP: non ovariectomized E2 (pg/ml) $19 \pm 2.3$ vs. $27,25 \pm 3, \mathrm{CRH}(\mathrm{pg} / \mathrm{ml})$ $3.6 \pm 0.6$ vs. $1.8 \pm 0.8$, ACTH $(\mathrm{pg} / \mathrm{ml}) 246.8 \pm 14.5$ vs. $161.2 \pm 7.9$, cortisol $(\mathrm{pg} / \mathrm{ml}) 28.4 \pm 4.5$ vs. $11.6 \pm 3.1$; Ovariectomized E2 (pg/ml) $5.06 \pm 2.1$ vs. $28.2 \pm 2.8$, CRH $(\mathrm{pg} / \mathrm{ml}) 4.9 \pm 2.1$ vs. $3.6 \pm 1$, ACTH $(\mathrm{pg} / \mathrm{ml}) 269.8 \pm 13.9$ vs. $163.2 \pm 7.9$, cortisol (pg/ml) $27.6 \pm 5.9$ vs. $12.7 \pm 3.1]$ [LI: non ovariectomized E2 (pg/ml) $15.9 \pm 0.9$ vs. $28.2 \pm 3$, CRH (pg/ml) $6.61 \pm 1.6$ vs $2.8 \pm 0.8$, ACTH (pg/ml) 266.7 \pm 7.3 vs. $162.2 \pm 7.9$, cortisol $(\mathrm{pg} / \mathrm{ml}) 35.6 \pm 6.5$ vs. $12.7 \pm$ 3.1; Ovariectomized E2 (pg/ml) $5.8 \pm 3.07$ vs. $27.8 \pm 3.2$, CRH (pg/ml) $6.9 \pm 1.8$ vs. $2.7 \pm 0.8$, ACTH (pg/ml) 300.1 \pm 10.4 vs. $161.7 \pm 6,6$, cortisol (pg/ml) $40.58 \pm 4.5$ vs. 11.5 \pm 2.7 ] (Tables 1 and 2).

However, estradiol replacement after ovariectomy succeded in inducing significant increase $(p<0.05)$ in the level of estradiol associated with significant decreases $(\mathrm{p}<0.05)$ in the levels of CRH, ACTH and cortisol in comparison to placebo treated ovariectomized female rats after systemic or local inflammation. [CLP: E2 (pg/ml) $33.5 \pm 5.3, \mathrm{CRH}(\mathrm{pg} / \mathrm{ml}) 1.4 \pm 0.8, \mathrm{ACTH}(\mathrm{pg} / \mathrm{ml}) 185.3 \pm$ 4.7, cortisol (pg/ml) $22.8 \pm 2.1$ ] [LI: E2 (pg/ml) $28 \pm 3.8$, CRH (pg/ml) $2.2 \pm 1.6$, ACTH (pg/ml) $190.5 \pm 6.4$, cortisol (pg/ml) 24.5 \pm 2.7 ] (Tables 1 and 2).

When we compared different CLP subgroups to corresponding subgroups subjected to LI, we noted an enhancement of HPA response during LI in comparison to CLP, in which the levels of CRH, ACTH and cortisol significantly increased $(\mathrm{p}<0.05)$. [CRH $(\mathrm{pg} / \mathrm{ml})$ : non ovariectomized $6.6 \pm 1.6$ vs. $3.6 \pm 0.6$, ovariectomized $6.9 \pm 1.8$ vs. $4.9 \pm 2.1$, estradiol treated ovariectomized $2.2 \pm 1.6$ vs. $1.4 \pm 0.8$; ACTH $(\mathrm{pg} / \mathrm{ml})$ : non ovariectomized $266.7 \pm 7.3$ vs. $246.8 \pm 14.5$, ovariectomized $300.1 \pm 10.4$ vs. $269.8 \pm 13.9$; Cortisol $(\mathrm{pg} / \mathrm{ml})$ : non ova- 
riectomized $35.6 \pm 6.5$ vs. $28.4 \pm 4.510$, ovariectomized $40.5 \pm 4.5$ vs. $27.6 \pm 5.9$, estradiol treated ovariectomized $24.5 \pm 2.7$ vs. $22.8 \pm 2.1$ ] (Figures 2 (a)-(c)).

\subsection{IL6 (Serum, Brain, Pituitary)}

We found that there were significant increases $(p<0.05)$

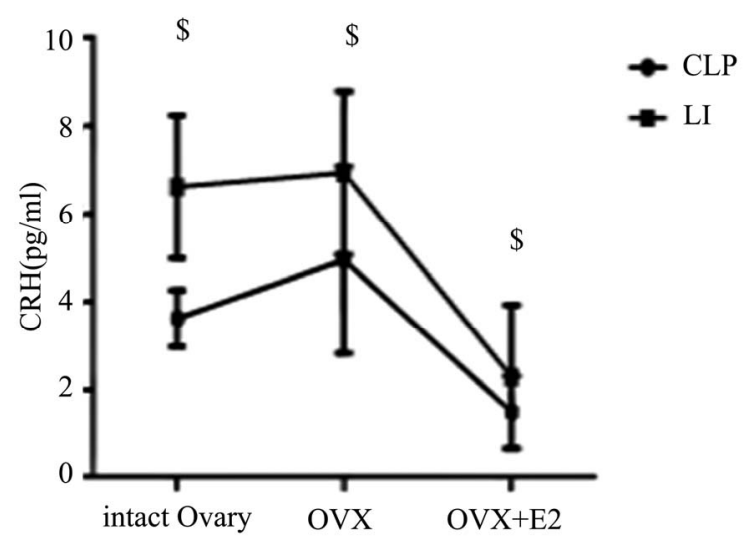

(a)

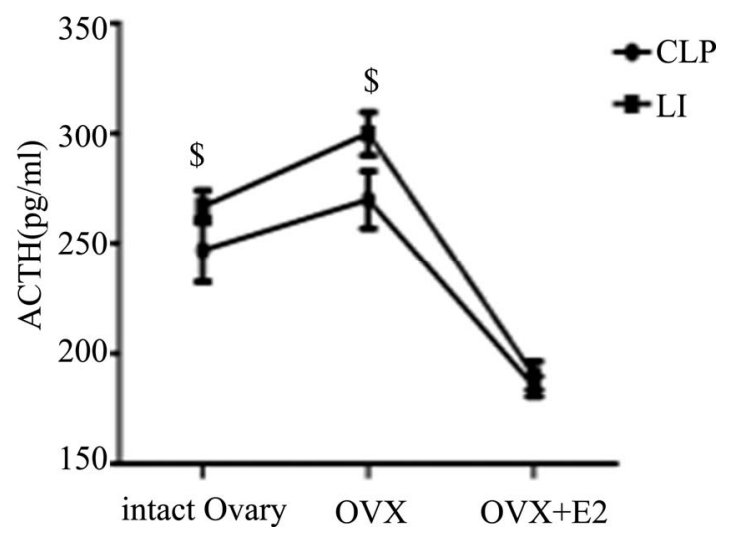

(b)

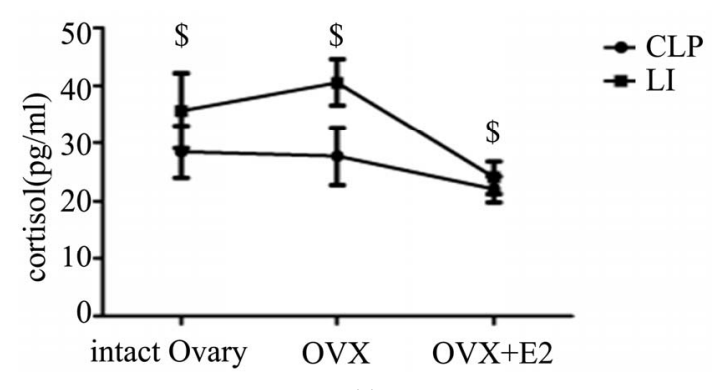

(c)

All values are expressed in means $\pm \mathrm{SD} .{ }^{\$} \mathrm{P}<0.05$ vs. corresponding CLP groups.

Figure 2. Evaluation of the effects of cecal ligation and puncture (CLP) and local inflammation (LI) on the plasma levels of corticotropin-releasing hormone (CRH) (A), adrenocorticotropes hormon (ACTH) (B) and cortisol $(\mathrm{C})$ in non ovariectomized, placebo treated ovariectomized $(\mathrm{OVX}+$ oil) and estradiol treated ovariectomized $(\mathrm{OVX}+\mathrm{E} 2)$ female rats. in serum IL6and pituitary Il6 in non ovariectomized and ovariectomized female rats subjected to either CLP or LI in comparison to corresponding sham subgroups [CLP: non ovariectomized serum IL6 $(\mathrm{ng} / \mathrm{ml}) 820.3 \pm 92$ vs. 517.7 \pm 151.9 , pituitary IL6 $(\mathrm{ng} / \mathrm{ml}) 273.3 \pm 29.1$ vs. $125.2 \pm 15.1$; Ovariectomized serum IL6 $(\mathrm{ng} / \mathrm{ml}) 865.1 \pm 51.5$ vs. $519.7 \pm$ 151.9, pituitaryIL6 $(\mathrm{ng} / \mathrm{ml}) 244.3 \pm 27.2$ vs. $127.2 \pm 15.1]$ [LI: non ovariectomized serum IL6 $(\mathrm{ng} / \mathrm{ml}) 864.8 \pm 94.9$ vs. $518.7 \pm 151.9$, pituitary IL6 $(\mathrm{ng} / \mathrm{ml}) 128.6 \pm 8.4$ vs. $126.2 \pm 15.1$; Ovariectomized serum IL6 (ng/ml) $919.2 \pm$ 297 vs. $513.2 \pm 151.2$, pituitary IL6 $(\mathrm{ng} / \mathrm{ml}) 257.5 \pm 26$ vs. $126.3 \pm 14.3$ ] (Tables 1 and 2).

In addition, we found that E2 replacemt after ovariectomy was associated with significant decreases $(p<0.05)$ in the levels of serum IL6, brain IL6 and pituitary I16 in comparison to placebo treated ovariectomized female rats after systemic or local inflammation. [Estradiol treated CLP: serum IL6 (ng/ml) $558 \pm 103.4$, brain IL6 $(\mathrm{ng} / \mathrm{ml}) 195.6 \pm 12.4$, pituitary IL6 $(\mathrm{ng} / \mathrm{ml}) 204.9 \pm 10.8]$ [Estradiol treated LI: serum IL6 $(\mathrm{ng} / \mathrm{ml}) 606.1 \pm 105$, brain IL6 $(\mathrm{ng} / \mathrm{ml}) 200.7 \pm 13.6$, pituitary IL6 $(\mathrm{ng} / \mathrm{ml})$ $207.4 \pm 8.3$ ] (Tables 1 and 2).

When we compared different CLP subgroups to corresponding subgroups subjected to LI, we found that there were significant increases $(p<0.05)$ in the level of brain and pituitary IL6 with local inflammation in com parison to CLP. [Brain IL6 ( $\mathrm{ng} / \mathrm{ml})$ : ovariectomized $288.7 \pm 7.7$ vs. $252.7 \pm 29.7$, estradiol treated ovariectomized $200.7 \pm 13.6$ vs. $195.6 \pm 12.4$; pituitary IL6 $(\mathrm{ng} / \mathrm{ml})$ : ovariectomized $257.5 \pm 26$ vs. $244.3 \pm 27.2$, estradiol treated ovariectomized $207.4 \pm 8.3$ vs. $204.9 \pm$ 10.8] (Figures 3 (a)-(c)).

\subsection{Nitrite (Brain, Pituitary)}

We found that there was significant increase $(p<0.05)$ in the brain nitrite level in non ovariectomized and ovariectomized female rats subjected to either CLP or LI in comparison to corresponding sham subgroups [CLP: non ovariectomized brain nitrite $(\mathrm{pg} / \mathrm{ml}) 271.3 \pm 24.5$ vs. 164 \pm 8.5 ; Ovariectomized brain nitrite $(\mathrm{pg} / \mathrm{ml}) 271.3 \pm 24.5$ vs $190.9 \pm 12.4$ ] [LI: non ovariectomized brain nitrite (pg/ml) $248.7 \pm 43.7$ vs. $165 \pm 8.5$; Ovariectomized brain nitrite (pg/ml) 258 vs. 24.7] (Tables 1 and 2).

In addition, we found that E2 replacemt after ovariectomy was associated with significant decreases $(p<0.05)$ in the levels of brain nitrite in comparison to placebo treated ovariectomized female rats after systemic or local inflammation. [Estradiol treated CLP: brain nitrite $(\mathrm{pg} / \mathrm{ml})$ $221.2 \pm 15.4$ ] [Estradiol treated LI: brain nitrite $(\mathrm{pg} / \mathrm{ml})$ $206 \pm 36.5]$ (Tables 1 and 2).

When we compared different CLP subgroups to corresponding subgroups subjected to $\mathrm{LI}$, we found that there was significant increase $(p<0.05)$ in the level of pituitary nitrite with local inflammation in comparison to 
Table 1. Evaluation of the levels of E2, CRH, ACTH, cortisol, IL6 and nitrite in sham CLP and all CLP subgroups.

\begin{tabular}{lccccc}
\hline & Sham CLP & ShamOVX + sham CLP & CLP & OVX+CLP & OVX+E2+CLP \\
\hline E2 (Pg/ml) & $27.2 \pm 3$ & $28.2 \pm 2.8$ & $19 \pm 2.3^{\$}$ & $5 \pm 2.1 !$ & $33.5 \pm 5.3^{\#}$ \\
CRH (pg/ml) & $1.8 \pm 0.8$ & $3.6 \pm 1$ & $3.6 \pm 0.6^{\S}$ & $4.9 \pm 2.1 !$ & $1.4 \pm 0.8^{\#}$ \\
ACTH (pg/ml) & $161.3 \pm 7.9$ & $163.3 \pm 7.9$ & $246.9 \pm 14.5^{\S}$ & $269.9 \pm 13.9 !$ & $185.3 \pm 4.7^{\#}$ \\
Cortisol (pg/ml) & $11.5 \pm 2.7$ & $12.7 \pm 3.1$ & $28.4 \pm 4.5^{\S}$ & $27.6 \pm 5.9^{\ddagger}$ & $22.8 \pm 2.1^{\#}$ \\
IL6 Serum (ng/ml) & $517.7 \pm 151.9$ & $519.7 \pm 151.9$ & $820.3 \pm 92^{\$}$ & $865.1 \pm 51.5^{!}$ & $558 \pm 103.4^{\#}$ \\
IL6 Pituitary (ng/ml) & $125.2 \pm 15.1$ & $127.2 \pm 15.1$ & $273.3 \pm 29.1^{\$}$ & $244.3 \pm 27.2^{!}$ & $204.9 \pm 10.8^{\#}$ \\
IL6 Brain (ng/ml) & $256.6 \pm 58.9$ & $258.4 \pm 59.1$ & $232.3 \pm 25$ & $252.7 \pm 29.7$ & $195.6 \pm 12.4^{\#}$ \\
Nitrite Pituitary (pg/ml) & $188.9 \pm 12.4$ & $166 \pm 8.5$ & $159.7 \pm 30.7$ & $265 \pm 28.2^{\sharp}$ & $226.9 \pm 20.2^{\#}$ \\
Nitrite Brain (pg/ml) & $164 \pm 8.5$ & $190.9 \pm 12.4$ & $232.1 \pm 15.3^{\$}$ & $271.3 \pm 24.5^{!}$ & $221.2 \pm 15.4^{\#}$ \\
\hline
\end{tabular}

E2: Estradiol, CRH: Corticotropin-releasing hormone, ACTH: Adrenocorticotropic Hormone, IL6: interleukin 6, CLP: cecal ligation and puncture, OVX: ovariectomy, The mean \pm SD. ${ }^{\$} \mathrm{P}<0.05$ vs. Sham CLP, ${ }^{\mathrm{P}}<0.05$ vs. Sham OVX+ sham CLP, ${ }^{\#} \mathrm{P}<0.05$ vs. OVX + CLP.

Table 2. Evaluation of the levels of E2, CRH, ACTH, cortisol, IL6 and nitrite in sham LI and all LI subgroups.

\begin{tabular}{cccccc}
\hline & Sham LI & $\begin{array}{c}\text { Sham OVX+ sham } \\
\text { LI }\end{array}$ & LI & OVX + LI & OVX+E2+LI \\
\hline E2 (Pg/ml) & $28.2 \pm 3$ & $27.8 \pm 3.2$ & $15.9 \pm 0.9^{\$}$ & $5.8 \pm 3^{!}$ & $28 \pm 3.8^{\#}$ \\
CRH (pg/ml) & $2.8 \pm 0.8$ & $2.7 \pm 0.8$ & $6.6 \pm 1.6^{\S}$ & $6.9 \pm 1.8^{!}$ & $2.2 \pm 1.6^{\#}$ \\
ACTH (pg/ml) & $162.3 \pm 7.9$ & $161.7 \pm 6.6$ & $266.7 \pm 7.3^{\S}$ & $300.1 \pm 10.4^{!}$ & $190.5 \pm 6.4^{\#}$ \\
Cortisol (pg/ml) & $12.7 \pm 3.1$ & $11.5 \pm 2.7$ & $35.6 \pm 6.5^{\$}$ & $40.5 \pm 4.5^{!}$ & $24.5 \pm 2.7^{\#}$ \\
IL6 Serum (ng/ml) & $518.7 \pm 151.9$ & $513.2 \pm 151.2$ & $864.8 \pm 94.9^{\S}$ & $919.2 \pm 297^{!}$ & $606.1 \pm 105^{\#}$ \\
IL6 Pituitary (ng/ml) & $126.2 \pm 15.1$ & $126.3 \pm 14.3$ & $128.6 \pm 8.4^{\S}$ & $257.5 \pm 26^{!}$ & $207.4 \pm 8.3^{\#}$ \\
IL6 Brain (ng/ml) & $257.4 \pm 59.1$ & $260.4 \pm 57.1$ & $230.9 \pm 18.3$ & $288.7 \pm 7.7$ & $200.7 \pm 13.6^{\#}$ \\
Nitrite Pituitary (pg/ml) & $189.9 \pm 12.4$ & $189.3 \pm 12.2$ & $244.8 \pm 39.1$ & $250.8 \pm 22.2$ & $250.2 \pm 22.5$ \\
Nitrite Brain (pg/ml) & $165 \pm 8.5$ & $163.2 \pm 10.2$ & $248.7 \pm 43.7^{\$}$ & $258 \pm 24.7^{!}$ & $206 \pm 36.5^{\#}$ \\
\hline
\end{tabular}

E2: Estradiol, CRH: Corticotropin-releasing hormone, ACTH:Adrenocorticotropic Hormone, IL6: interleukin 6, LI: local inflammation, OVX: ovariectomy. The mean \pm SD. ${ }^{\$} \mathrm{P}<0.05$ vs. Sham $\mathrm{LI},{ }^{!} \mathrm{P}<0.05$ vs. Sham OVX+ sham $\mathrm{LI},{ }^{\#} \mathrm{P}<0.05$ vs. OVX+LI

CLP. [Pituitary nitrite $(\mathrm{pg} / \mathrm{ml})$ : non ovariectomized 244.8 \pm 39.1 vs. $159.7 \pm 30.7$, estradiol treated ovariectomized $250.2 \pm 22.5$ vs. $226.9 \pm 20.2]$ (Figure 4 (a)-(b)).

\section{DISCUSSION}

In our study, we found that both the level of estradiol hormone and the type of inflammation (stress) could play an important role in the degree of HPA response. Regarding the level of estradiol hormone, we found that in both CLP and LI the reduced level of estradiol induced by stress (inflammation) or surgical ovariectomy was associated with increases in the plasma levels of CRH, ACTH and Cortisol. Meanwhile, estradiol replacement after ovariectomy induced significant increase in estradiol to certain physiological level, which was associated with significant decreases in the plasma levels of $\mathrm{CRH}$,

\section{ACTH and Cortisol.}

Previous studies demonstrated that the stress released hormones such as $\mathrm{CRH}$, vasopressin and glucocorticoids could inhibit the gonadal steroid production [25]. With low level of estradiol the other inflammatory mediators take the upper hand over sex hormones on the HPA response resulting in stimulatory effects at the level of hypothalamic CRF release or directly on the pituitary itself to stimulate ACTH secretion [26,27]. In addition, previous studies demonstrated that castration enhances the ACTH and corticosterone response to IL- $1 \beta$ and lipopolysaccharide (LPS) and this was reversed by E2 replacement [28-31]. However, our study differ in that we used not only CLP model, which appears to be the best existing alternative for emulating human polymicrobial sepsis to date and could mimic the pathophysiologic 


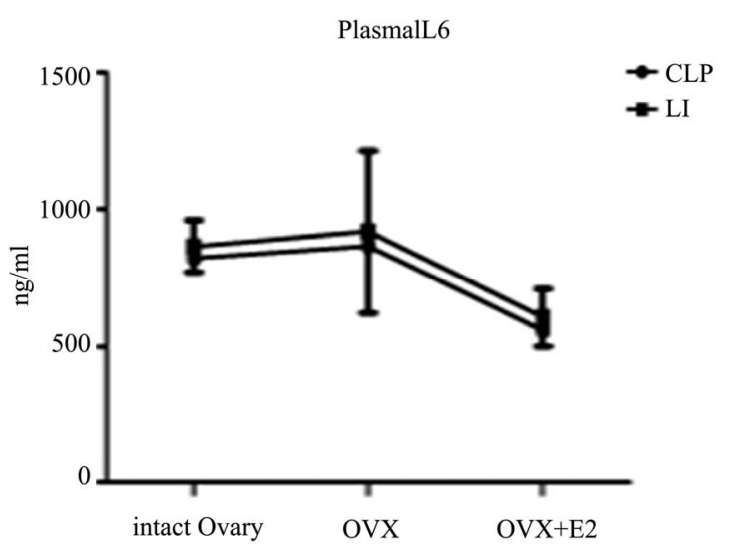

(a)

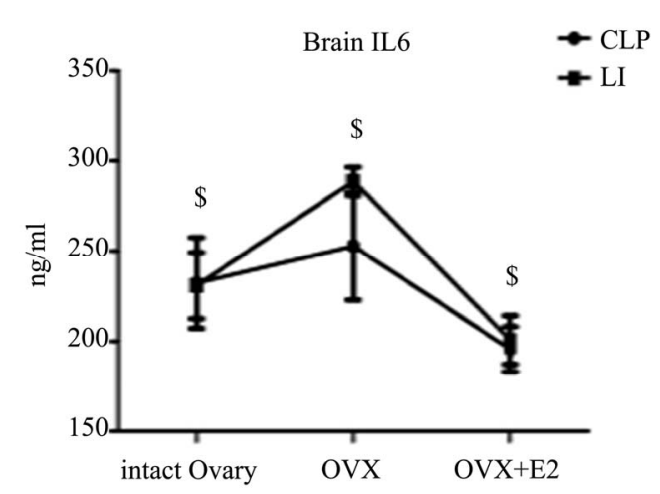

(b)

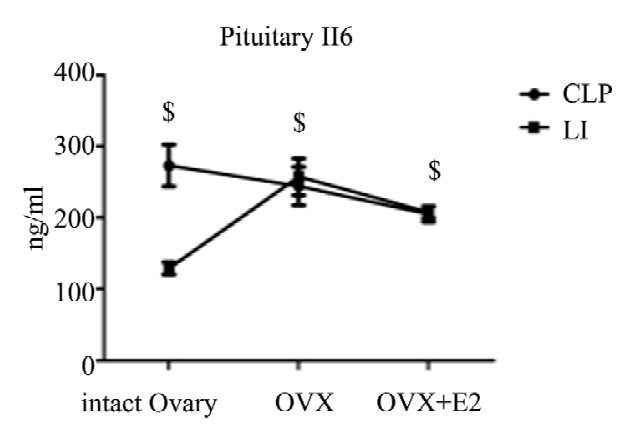

(c)

All values are expressed in means $\pm \mathrm{SD}$. ${ }^{\mathrm{S}} \mathrm{P}<0.05$ vs. corresponding CLP groups.

Figure 3. Evaluation of the effects of cecal ligation and puncture (CLP) and local inflammation(LI) on the plasma level of interleukin 6 (IL6) (A), the brain level of IL6 (B) and the pituitary level of IL6 (C) in non ovariectomized, placebo treated ovariectomized $(\mathrm{OVX}+\mathrm{oil})$ and estradiol treated ovariectomized $(\mathrm{OVX}+\mathrm{E} 2)$ female rats.

consequences of sepsis [32,33], but also local inflammation as many studies have been largely restricted to the HPA response to LPS. However, it is doubtful that LPS activation of the HPA axis can be viewed as a general model by which infectious/inflammatory insults influence neuroendocrine activity [34]. Another difference inour studies is that we evaluated the HPA response by the measurement of the plasma levels of $\mathrm{CRH}, \mathrm{ACTH}$

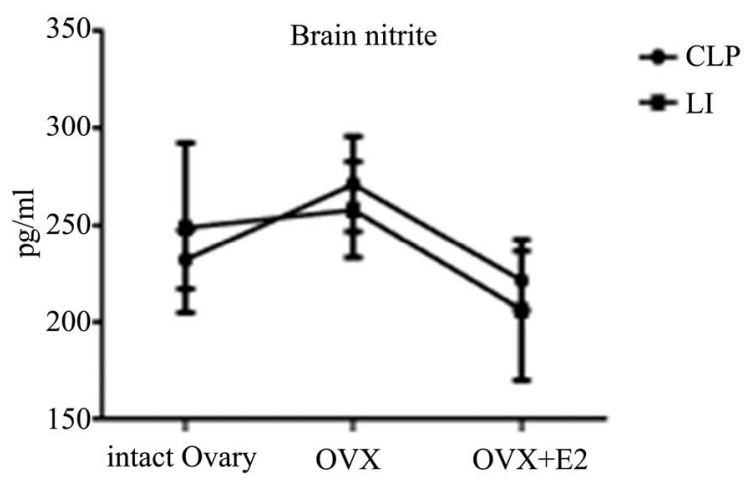

(a)

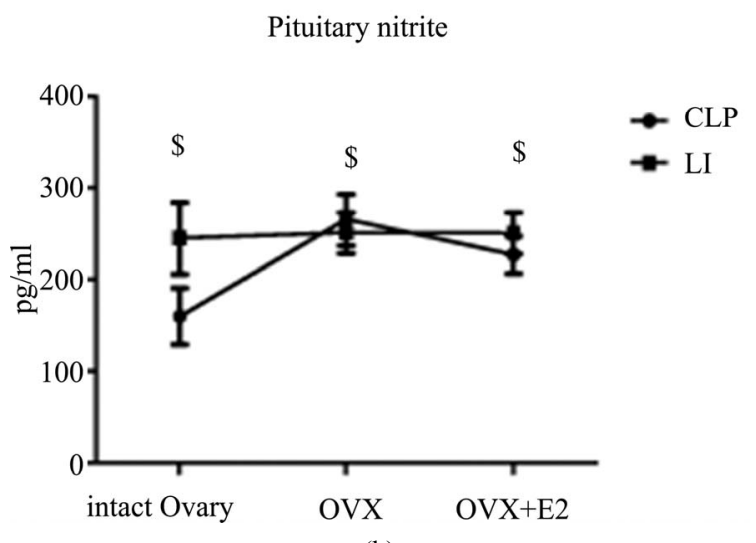

(b)

All values are expressed in means $\pm \mathrm{SD} .{ }^{\$} \mathrm{P}<0.05$ vs. corresponding CLP groups.

Figure 4. Evaluation of the effects of cecal ligation and puncture (CLP) and local inflammation (LI) on the brain level of nitrite (A) and the pituitary level of nitrite (B) in non ovariectomized, placebo treated ovariectomized (OVX + oil) and estradiol treated ovariectomized (OVX + E2) female rats.

and cortisol instead of the measurement of the tissue content of CRH and ACTH, which may not well reflect the rapidly releasable pool of these hormones, which is the more important parameter in acute hormonal responses. This is because tissue contents of hormones, in general, represent a difference between an increase by synthesis and a decrease by release, which thus raises the possibility that similar hormonal contents in different experimental groups could result if both the synthesis and release are altered to a similar degree but in an opposite direction $[28,30,35,36]$.

In contrast to our study, Watanobe and Yoneda [35] found that OVX significantly diminished the LPS-induced ACTH release, and that this effect was completely abolished by supplementing $E_{2}$ suggesting that estrogen actually promotes inflammatory induced stress response. In addition, Nappi et al. [37] reported that CRH gene was highly expressed in the parvocellular subdivision of the paraventricular nucleus during the morning of proes- 
trus (higher sex hormones) compared with diestrous females (lower sex hormones) subjected to systemic injection of LPS. Lunga and Herbert [38] showed also that basal and stress-activated corticosterone levels have been shown to vary across the oestrous cycle, being at their maximal during the proestrous phase when circulating E2 levels are high. Those discrepancies between our results and previous results could be due to differences in experimental circumstances, species and the concentration of estradiol reached after ovariectomy and achieved by treatment.

To our knowledge, our study is the first that compare the effects of CLP and LI on HPA response with different estradiol levels. We found that LI is associated with enhancement of HPA response in comparison to CLP. This could suggest that mechanism involved in HPA enhancement differ between LI and CLP. This could support both Mannino et al. and Turnbull and Rivier [27-39], who showed that the immediate response to intra-plantar formalin (phase 1) results from the stimulation of primary afferent nociceptors, whereas the latter response, phase 2, is generated by a reduced but continuing stimulation of peripheral nociceptors, and activation of inflammatory mediators, and that both phases have an effect on the HPA response during local inflammation. For this reason, our second aim was to measure central and peripheral formed IL6, which plays a major role in endotoxin-induced stimulation of the HPA axis, as indicated by the fact that antibodies against IL6 almost completely block the HPA response to endotoxin [40-42], and central formed nitrite (as an indicator of NO production). We wanted to see if there are differences in IL6 and NO production with different estradiol levels and different type of inflammation that could explain the previous observed differences in HPA responses. We found that low physiological level of estradiol induced by stress and surgical ovariectomy associated with significant increases in serum and pituitary IL6 both in LI and CLP. However, estradiol replacement after ovariectomy succeded to increase estradiol to certain physiological level associated with significant lowering of serum, pituitary and brain IL6.

Our finding support previous studies, which showed relation between estradiol and IL6, such as Chiu et al. [43], who observed a negative correlation between circulating IL6 and E2 concentrations during the normal menstrual cycle. Moreover, Puder et al. [44] demonstrated that E2 attenuates the endotoxin-induced stimulation of IL6, and TNF-alpha release in the postmenopausal women.

The exact mechanisms by which estrogen interferes with cytokine activity are still incompletely known but may potentially include interactions of the estrdiol receptor (ER) with other transcription factors, modulation of IL6 promoter and nitric oxide activity, an anti-oxidative effect, a plasma membrane action, and changes in immune cell function[45,46].
In addition, we found that low physiological level of estradiol associated with significant increases in the brain nitrite, and significant lowering of the brain nitrite was achieved after estradiol replacement. These findings could suggest the enhancement of NO induced stimulation of HPA axis during inflammation, as indicated by Puder et al. [44], who suggested that the endogenous NO seems to restrain the HPA response to inflammatory stimuli. Uribe et al. [47] found also a temporal correlation between endotoxin-induced activation of the HPA axis and stimulation of neuronal NO synthase in the paraventricular nucleus of the hypothalamus. Although, previous studies demonstrated the inhibitory effect of estrogen on NO productions $[45,48]$ our study is the first to our knowledge that showed the inhibitory effect of high physiological level of estradiol on NO production during local and systemic inflammation. The mechanism by which estradiol has differential effects on the different NO synthase isoforms is not well understood. Both nuclear and extra-nuclear pathways are used by estradiol to affect the NOS isoforms. However, the inhibitory effect of estradiol on iNOS may be due to the inhibition of cytokines production [49].

We found also significant differences between CLP and LI in central produced nitrite and IL6 (brain and pituitary), with higher production associated with LI. This finding could partial explain the previous noted LI induced higher HPA response and support the important role of central formed NO and Il6 on HPA response during local inflammation. However, we could not exclude the involvement of other factors such as the nociceptive afferent fiber $[8,34]$ in the differences between local inflammation and systemic inflammation induced HPA axis responses. However, we were surprised that central rather than peripheral formed IL6 showed significant difference during local inflammation, this observation raise an important question that need further investigation: whether the peripheral inflammatory mediators or the neural afferent stimulation is responsible for the observed enhanced central inflammation during local inflammation?

\section{CONCLUSION}

From our study, we suggest that both estradiol level and the type of stress are important determinant factors for HPA response, and their effects on HPA could be mediated through IL6 and NO. However, further molecular study will provide additional mechanistic information and further support for the conclusions derived in this study.

\section{ACKNOWLEDGEMENTS}

The skillful technical assistance of Afaf, Aza and Tarek is appreci- 
ated.

\section{REFERENCES}

[1] Bone, R.C. (1992) Toward an epidemiology and natural history of SIRS (systemic inflammatory response syndrome). The Journal of the American Medical Association, 268, 3452-3455. doi:10.1001/jama.1992.03490240060037

[2] Brun-Buisson, C. (2000) The epidemiology of the systemic inflammatory response. Intensive Care Medicine, 26, S64-S74. doi:10.1007/s001340051121

[3] Wade, S., Bussow, M. and Hanisch, E. (1998) Epidemiology of systemic inflammatory response syndrome, infection and septic shock in surgical intensive care patients. Der Chirurg, 69, 648-655. doi:10.1007/s001040050470

[4] Van den Berghe, G., de Zegher, F. and Bouillon, R. (1998) Clinical review 95: Acute and prolonged critical illness as different neuroendocrine paradigms. The Journal of Clinical Endocrinology \& Metabolism, 83, 1827-1834. doi:10.1210/jc.83.6.1827

[5] Mebis, L., Debaveye, Y., Ellger, B., Derde, S., Ververs, E.J., et al. (2009) Changes in the central component of the hypothalamus-pituitary-thyroid axis in a rabbit model of prolonged critical illness. Critical Care, 13, R147. doi:10.1186/cc8043

[6] Sriram, K., Rodriguez-Fernandez, M. and Doyle, F.J. III, (2012) Modeling cortisol dynamics in the neuro-endocrine axis distinguishes normal, depression, and posttraumatic stress disorder (PTSD) in humans. PLoS Computational Biology, 8, e1002379.

doi:10.1371/journal.pcbi.1002379

[7] Tsigos, C. and Chrousos, G.P. (2002) Hypothalamic-pituitary-adrenal axis, neuroendocrine factors and stress. Journal of Psychosomatic Research, 53, 865-871. doi:10.1016/S0022-3999(02)00429-4

[8] Turnbull, A.V. and Rivier, C.L. (1999) Regulation of the hypothalamic-pituitary-adrenal axis by cytokines: Actions and mechanisms of action. Physiological Reviews, 79, $1-71$.

[9] Leon, L.R., White, A.A. and Kluger, M.J. (1998) Role of IL-6 and TNF in thermoregulation and survival during sepsis in mice. American Journal of Physiology. Regulatory, Integrative and Comparative Physiology, 275, R269R277.

[10] Zellweger, R., Wichmann, M.W., Ayala, A., Stein, S., DeMaso, C.M., et al. (1997) Females in proestrus state maintain splenic immune functions and tolerate sepsis better than males. Critical Care Medicine, 25, 106-110. doi:10.1097/00003246-199701000-00021

[11] Jankord, R., Turk, J.R., Schadt, J.C., Casati, J., Ganjam, V.K., et al. (2007) Sex difference in link between interleukin-6 and stress. Endocrinology, 148, 3758-3764. doi:10.1210/en.2006-1650

[12] Serova, L.I., Harris, H.A., Maharjan, S. and Sabban, E.L. (2010) Modulation of responses to stress by estradiol benzoate and selective estrogen receptor agonists. Journal of Endocrinology, 205, 253-262.
doi:10.1677/JOE-10-0029

[13] Evuarherhe, O., Leggett, J., Waite, E., Kershaw, Y. and Lightman, S. (2009) Reversal of the hypothalamo-pituitary-adrenal response to oestrogens around puberty. Journal of Endocrinology, 202, 279-285. doi:10.1677/JOE-09-0175

[14] Weiser, M.J. and Handa, R.J. (2009) Estrogen impairs glucocorticoid dependent negative feedback on the hypothalamic-pituitary-adrenal axis via estrogen receptor alpha within the hypothalamus. Neuroscience, 159, 883895. doi:10.1016/j.neuroscience.2008.12.058

[15] Kumsta, R., Entringer, S., Koper, J.W., Van Rossum, E.F., Hellhammer, D.H., et al. (2007) Sex specific associations between common glucocorticoid receptor gene variants and hypothalamus-pituitary-adrenal axis responses to psychosocial stress. Biological Psychiatry, 62, 863-869. doi:10.1016/j.biopsych.2007.04.013

[16] Windle, R.J., Gamble, L.E., Kershaw, Y.M., Wood, S.A., Lightman, S.L., et al. (2006) Gonadal steroid modulation of stress-induced hypothalamo-pituitary-adrenal activity and anxiety behavior: Role of central oxytocin. Endocrinology, 147, 2423-2431. doi:10.1210/en.2005-1079

[17] Seale, J.V., Wood, S.A., Atkinson, H.C., Harbuz, M.S. and Lightman, S.L. (2004) Gonadal steroid replacement reverses gonadectomy-induced changes in the corticosterone pulse profile and stress-induced hypothalamic-pituitary-adrenal axis activity of male and female rats. Journal of Neuroendocrinology, 16, 989-998. doi:10.1111/j.1365-2826.2004.01258.x

[18] Isgor, C., Cecchi, M., Kabbaj, M., Akil, H. and Watson, S.J. (2003) Estrogen receptor beta in the paraventricular nucleus of hypothalamus regulates the neuroendocrine response to stress and is regulated by corticosterone. $\mathrm{Neu}$ roscience, 121, 837-845. doi:10.1016/S0306-4522(03)00561-X

[19] McCormick, C.M., Linkroum, W., Sallinen, B.J. and Miller, N.W. (2002) Peripheral and central sex steroids have differential effects on the HPA axis of male and female rats. Stress, 5, 235-247. doi:10.1080/1025389021000061165

[20] Kirschbaum, C., Kudielka, B.M., Gaab, J., Schommer, N.C. and Hellhammer, D.H. (1999) Impact of gender, menstrual cycle phase, and oral contraceptives on the activity of the hypothalamus-pituitary-adrenal axis. Psychosomatic Medicine, 61, 154-162.

[21] Suzuki, S. and Handa, R.J. (2004) Regulation of estrogen receptor-beta expression in the female rat hypothalamus: differential effects of dexamethasone and estradiol. Endocrinology, 145, 3658-3670. doi:10.1210/en.2003-1688

[22] Deng, J., Muthu, K., Gamelli, R., Shankar, R. and Jones, S.B. (2004) Adrenergic modulation of splenic macrophage cytokine release in polymicrobial sepsis. American journal of physiology. Cell physiology, 287, C730-C736. doi:10.1152/ajpcell.00562.2003

[23] Green, P.G., Dahlqvist, S.R., Isenberg, W.M., Strausbaugh, H.J., Miao, F.J., et al. (1999) Sex steroid regulation of the inflammatory response: Sympathoadrenal dependence in the female rat. The Journal of Neuroscience, 19, 40824089 . 
[24] Malmberg, A.B. and Yaksh, T.L. (1995) Cyclooxygenase inhibition and the spinal release of prostaglandin E2 and amino acids evoked by paw formalin injection: A microdialysis study in unanesthetized rats. The Journal of Neuroscience, 15, 2768-2776.

[25] Roy, B.N., Reid, R.L. and Van Vugt, D.A. (1999) The effects of estrogen and progesterone on corticotropin-releasing hormone and arginine vasopressin messenger ribonucleic acid levels in the paraventricular nucleus and supraoptic nucleus of the rhesus monkey. Endocrinology, 140, 2191-2198. doi:10.1210/en.140.5.2191

[26] Berkenbosch, F., van Oers, J., Del Rey, A., Tilders, F. and Besedovsky, H. (1987) Corticotropin-releasing factorproducing neurons in the rat activated by interleukin-1. Science, 238, 524-526. doi:10.1126/science.2443979

[27] Turnbull, A.V. and Rivier, C. (1996) Corticotropin-releasing factor, vasopressin, and prostaglandins mediate, and nitric oxide restrains, the hypothalamic-pituitary-adrenal response to acute local inflammation in the rat. Endocrinology, 137, 455-463. doi:10.1210/en.137.2.455

[28] Rivier, C. (1994) Stimulatory effect of interleukin-1 beta on the hypothalamic-pituitary-adrenal axis of the rat: Influence of age, gender and circulating sex steroids. Journal of Endocrinology, 140, 365-372. doi:10.1677/joe. 0.1400365

[29] Lee, S. and Rivier, C. (1995) Altered ACTH and corticosterone responses to interleukin-1 beta in male rats exposed to an alcohol diet: Possible role of vasopressin and testosterone. Alcoholism: Clinical and Experimental Research, 19, 200-208. doi:10.1111/j.1530-0277.1995.tb01493.x

[30] Xiao, E.N., Xia-Zhang, L., Ferin, M. and Wardlaw, S.L. (2001) Differential effects of estradiol on the adrenocorticotropin responses to interleukin-6 and interleukin-1 in the monkey. Endocrinology, 142, 2736-2741. doi:10.1210/en.142.7.2736

[31] Spinedi, E., Suescun, M.O., Hadid, R., Daneva, T. and Gaillard, R.C. (1992) Effects of gonadectomy and sex hormone therapy on the endotoxin-stimulated hypothalamo-pituitary-adrenal axis: Evidence for a neuroendocrine-immunological sexual dimorphism. Endocrinology, 131, 2430-2436. doi:10.1210/en.131.5.2430

[32] Hubbard, W.J., Choudhry, M., Schwacha, M.G., Kerby, J.D., Rue, L.W. III, et al. (2005) Cecal ligation and puncture. Shock, 24, 52-57. doi:10.1097/01.shk.0000191414.94461.7e

[33] Maier, S., Traeger, T., Entleutner, M., Westerholt, A., Kleist, B., et al. (2004) Cecal ligation and puncture versus colon ascendens stent peritonitis: Two distinct animal models for polymicrobial sepsis. Shock, 21, 505-511. doi:10.1097/01.shk.0000126906.52367.dd

[34] Hopkins, S.J. (2007) Central nervous system recognition of peripheral inflammation: A neural, hormonal collaboration. Acta Bio-Medica: Atenei Parmensis, 78, 231-247.

[35] Watanobe, H. and Yoneda, M. (2003) A mechanism underlying the sexually dimorphic ACTH response to lipopolysaccharide in rats: Sex steroid modulation of cytokine binding sites in the hypothalamus. The Journal of
Physiology, 547, 221-232.

doi:10.1113/jphysiol.2002.032169

[36] Watanobe, H., Anzai, J., Nigawara, T., Habu, S. and Takebe, K. (1996) Effects of gender and gonadectomy on $\mathrm{ACTH}$ response to interleukin-1beta in the rat: Comparison with the modulation of ACTH response to immobilization stress. Neuroimmunomodulation, 3, 254-258.

[37] Nappi, R.E., Bonneau, M.J. and Rivest, S. (1997) Influence of the estrous cycle on c-fos and $\mathrm{CRH}$ gene transcription in the brain of endotoxin-challenged female rats Neuroendocrinology, 65, 29-46. doi:10.1159/000127162

[38] Lunga, P. and Herbert, J. (2004) 17Beta-oestradiol modulates glucocorticoid, neural and behavioural adaptations to repeated restraint stress in female rats. Journal of Endocrinology, 16, 776-785. doi:10.1111/j.1365-2826.2004.01234.x

[39] Mannino, C.A., South, S.M., Quinones-Jenab, V. and Inturrisi, C.E. (2007) Estradiol replacement in ovariectomized rats is antihyperalgesic in the formalin test. The Journal of Pain, 8, 334-342. doi:10.1016/j.jpain.2006.10.002

[40] Kasckow, J.W., Regmi, A., Gill, P.S., Parkes, D.G. and Geracioti, T.D. (1997) Regulation of corticotropin-releasing factor (CRF) messenger ribonucleic acid and CRF peptide in the amygdala: Studies in primary amygdalar cultures. Endocrinology, 138, 4774-4782. doi:10.1210/en.138.11.4774

[41] Langlais, D., Couture, C., Balsalobre, A. and Drouin, J. (2008) Regulatory network analyses reveal genome-wide potentiation of LIF signaling by glucocorticoids and define an innate cell defense response. PLoS Genetics, 4, e1000224. doi:10.1371/journal.pgen.1000224

[42] Raber, J., O'Shea, R.D., Bloom, F.E. and Campbell, I.L. (1997) Modulation of hypothalamic-pituitary-adrenal function by transgenic expression of interleukin- 6 in the CNS of mice. The Journal of Neuroscience, 17, 94739480 .

[43] Chiu, K.M., Arnaud, C.D., Ju, J., Mayes, D., Bacchetti, P., et al. (2000) Correlation of estradiol, parathyroid hormone, interleukin-6, and soluble interleukin-6 receptor during the normal menstrual cycle. Bone, 26, 79-85. doi:10.1016/S8756-3282(99)00243-4

[44] Puder, J.J., Freda, P.U., Goland, R.S. and Wardlaw, S.L. (2001) Estrogen modulates the hypothalamic-pituitaryadrenal and inflammatory cytokine responses to endotoxin in women. The Journal of Clinical Endocrinology \& Metabolism, 86, 2403-2408. doi:10.1210/jc.86.6.2403

[45] Pfeilschifter, J., Koditz, R., Pfohl, M. and Schatz, H. (2002) Changes in proinflammatory cytokine activity after menopause. Endocrine Reviews, 23, 90-119. doi:10.1210/er.23.1.90

[46] Bondeson, J., Foxwell, B., Brennan, F. and Feldmann, M. (1999) Defining therapeutic targets by using adenovirus: Blocking NF-kappaB inhibits both inflammatory and destructive mechanisms in rheumatoid synovium but spares anti-inflammatory mediators. Proceedings of the National Academy of Sciences of the United States of America, 96, 5668-5673. doi:10.1073/pnas.96.10.5668 
[47] Uribe, R.M., Lee, S. and Rivier, C. (1999) Endotoxin stimulates nitric oxide production in the paraventricular nucleus of the hypothalamus through nitric oxide synthase I: Correlation with hypothalamic-pituitary-adrenal axis activation. Endocrinology, 140, 5971-5981.

doi:10.1210/en.140.12.5971

[48] Vegeto, E., Bonincontro, C., Pollio, G., Sala, A., Viappiani, S., et al. (2001) Estrogen prevents the lipopolysac- charide-induced inflammatory response in microglia. The Journal of Neuroscience, 21, 1809-1818.

[49] Turgeon, J.L., Carr, M.C., Maki, P.M., Mendelsohn, M.E. and Wise, P.M. (2006) Complex actions of sex steroids in adipose tissue, the cardiovascular system, and brain: Insights from basic science and clinical studies. Endocrine Reviews, 27, 575-605. doi:10.1210/er.2005-0020 